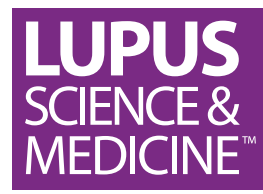

To cite: Liu Y, Chen S, Yang G, et al. ANA-positive primary immune thrombocytopaenia: a different clinical entity with increased risk of connective tissue diseases. Lupus Science \& Medicine 2021;8:e000523. doi:10.1136/ lupus-2021-000523

- Additional supplemental material is published online only. To view, please visit the journal online (http://dx.doi.org/10. 1136/lupus-2021-000523).

YL, SC and GY contributed equally.

Received 27 May 2021 Accepted 16 September 2021

Check for updates

(c) Author(s) (or their employer(s)) 2021. Re-use permitted under CC BY-NC. No commercial re-use. See rights and permissions. Published by BMJ.

${ }^{1}$ Department of Rheumatology and Clinical Immunology,

The First Affiliated Hospital of

Xiamen University, Xiamen,

Fujian, China

${ }^{2}$ School of Medicine, Xiamen University, Xiamen, China

${ }^{3}$ Department of Rheumatology, The Affiliated Hospital of Southwest Medical University, Luzhou, China

Correspondence to

Dr Guixiu Shi; gshi@xmu.edu.cn

\title{
ANA-positive primary immune thrombocytopaenia: a different clinical entity with increased risk of connective tissue diseases
}

\author{
Yuan Liu (10 , ${ }^{1,2}$ Shiju Chen, ${ }^{1,2}$ Guomei Yang, ${ }^{2,3}$ Bin Wang, ${ }^{1}$ Jinying Lan, ${ }^{1,2}$ Fan Dai, ${ }^{1,2}$ \\ Peishi Rao, ${ }^{1,2}$ Puqi Wu, ${ }^{1,2}$ Hongyan Qian, ${ }^{1,2}$ Guixiu Shi (i) ${ }^{1,2}$
}

\begin{abstract}
Objective Primary immune thrombocytopaenia (ITP) is highly heterogeneous. ANA-positive primary ITP may resemble the preclinical stage of connective tissue diseases (CTDs), but is still considered primary ITP due to a controversial CTD risk assessment in this group. The objective of this study was to clarify the risk of CTD in ANA-positive patients with primary ITP.

Methods We performed a retrospective cohort study and a meta-analysis. 586 patients with newly diagnosed primary ITP were followed up and Cox regression analyses were used to analyse the associations of ANA positivity and other immune parameters with CTD development. Results The mean follow-up time was 37 (19-56) months. ANA was positive in $21.33 \%$ (125 of 586 ) of patients with primary ITP in our retrospective cohort, and the overall rate of ANA positivity in the meta-analysis was $17.06 \%$ (369 of 2163). The adjusted HR for CTD in ANA-positive primary ITP was $6.15(95 \% \mathrm{Cl} 2.66$ to

Key messages
What is already known about this subject?
Patients with primary immune thrombocytopaenia
(ITP) are a heterogeneous population, but little is
known about the subclassification of primary ITP
clinically.
What does this study add?
ANA-positive primary ITP is a distinct subgroup of
primary ITP associated with a high risk of developing
connective tissue diseases (CTDs), especially SLE.
How might this impact on clinical practice or future
developments?
This study proposes a prediction model which might
assist clinicians in identifying individual patients with
primary ITP at high risk of CTD especially in the first
4 years after the first established ITP diagnosis.
\end{abstract}
$14.23, p<0.001)$. Five patients in the ANA-positive group developed SLE (5 of 125, 4.0\%), significantly higher than in the ANA-negative group ( 0 of $461,0 \%$ ). A clinical model combining ANA, anti-Sjogren's syndrome A antibody and C3 was successfully developed to predict the risk of CTD in patients with primary ITP. Increased risk of CTD (risk ratio $=12.43,95 \% \mathrm{Cl} 7.91$ to $19.55, \mathrm{p}<0.00001$ ), especially SLE (risk ratio $=30.41,95 \% \mathrm{Cl} 13.23$ to $69.86, p<0.00001$ ), among ANA-positive patients with primary ITP was confirmed by a meta-analysis of previous studies and the present study.

Conclusions The findings suggest that ANA-positive primary ITP is a clinical entity distinct from other primary ITPs and is associated with increased risk of developing CTDs, especially SLE.

\section{INTRODUCTION}

Immune thrombocytopaenia (ITP) is characterised by a platelet count of $<100 \times 10^{9} / \mathrm{L}$ due to an immune destruction of platelets and is classified as primary or secondary ITP based on the presence of underlying diseases. ${ }^{1}$ Primary ITP is a diagnosis of exclusion with great heterogeneity in its pathogenesis and clinical outcomes. ${ }^{2}$ However, little is known about the subsets of primary ITP and the best targeted therapy for patients with primary ITP.

Positive ANA is a distinct characteristic of connective tissue diseases (CTDs), including SLE, Sjogren's syndrome, antiphospholipid syndrome (APS) and undifferentiated connective tissue diseases (UCTD). It has been well recognised that ANA can be detected in some patients with primary ITP who do not meet the diagnostic criteria for any defined CTD. ${ }^{3}$ Thus, it has been proposed that positive-ANA primary ITP might be a distinct subset of primary ITP due to the risk of developing CTD following the first standardisation guideline for ITP in 2009. ${ }^{4}$ However, current standard still considers these patients to have primary ITP, and the treatment strategy for these patients is similar to those with other primary ITPs due to the controversial results of studies assessing the risk of CTD. ${ }^{3}$

The presence of positive ANA and thrombocytopaenia are both closely associated with 
CTDs, especially with SLE. Patients with ANA-positive primary ITP may resemble the preclinical stage of SLE in some situations. It is sometimes struggling for a rheumatologist to make the most appropriate treatment strategy for these patients because the principles in SLE and primary ITP treatment are quite different especially in terms of maintenance therapy. Their prognosis and impact on quality of life are also quite different.

To appropriately assess the similarities and differences between ANA-positive primary ITP and other primary ITPs, it is necessary to comprehensively clarify the impact of ANA positivity on the prognosis of ITP. Hence, we conducted a retrospective cohort study on the risk of CTD in ANA-positive primary ITP and additionally performed a meta-analysis on previous studies as well as the present study to assess the overall risk of CTD development in ANA-positive patients with primary ITP. We also explored potential factors associated with risk of CTD development in ANA-positive patients with primary ITP.

\section{METHODS}

\section{Study participants}

This study was a retrospective cohort study assessing the risk of CTD in patients with primary ITP with positive ANA. Informed consent to publish was obtained.

The study design is shown in online supplemental figure 1. Patients with isolated thrombocytopaenia as the first clinical manifestation and were diagnosed with primary ITP in both the inpatient and outpatient departments of our hospital between 2014 and 2020 were reviewed. The inclusion criterion was primary ITP. The criteria for diagnosis of primary ITP were based on the International Working Group (IWG) guidelines. ${ }^{15}$ The exclusion criteria were as follows: infections, severe hepatic and renal insufficiency, autoimmune thyroid disease, other haematological diseases, cancer, active hepatitis, liver cirrhosis, hypersplenism, gestational thrombocytopaenia, pseudo-thrombocytopaenia, drug-induced thrombocytopaenia, CTDs, repeated hospitalisation and without data on immune tests. A total of 803 patients with primary ITP with available data on autoantibody profile were included in this study. Of these, 217 patients were lost to follow-up. There were no differences in characteristics, including age, gender, ANA positivity, platelet count or extractable nuclear antigen antibody (ENA) positivity, between ITP populations who were successfully followed up and those who failed follow-up (online supplemental table 1).

\section{Assessment of CTD development and relevant variables}

Participants were followed up by telephone interview from the time they were diagnosed with ITP. An identical, detailed questionnaire on the presence of symptoms related to CTDs, including fever, arthralgias, joint swelling, joint deformity, Raynaud's phenomenon, etc, as well as the treatment regimen and response to treatment, was administered to each patient during the interview. Patients who met the diagnostic criteria for CTDs (including SLE, primary Sjogren's syndrome (pSS), APS, UCTD, etc) were defined as the main outcome of this study. The diagnostic criteria for CTDs were according to the guidelines of ACR (American College of Rheumatology)/EULAR (European Alliance of Associations for Rheumatology) ${ }^{6-11}$ Baseline information of each patient at the time of ITP diagnosis, including age, sex, clinical manifestations and laboratory findings such as platelet level, was recorded and analysed. Severity of bleeding manifestations at onset was assessed by the ITP Bleeding Scale. ${ }^{12}$ Resistance to steroids was defined as refractory to glucocorticoid (GC) treatment with a platelet count remaining $<30 \times 10^{9} / \mathrm{L}$ or less than a twofold increase from baseline platelet count, or bleeding, or dependence on GC following 4 weeks of treatment. Immunological tests including presence of ANA, anti-dsDNA (double strand DNA) antibody, ENA (EUROIMMUN, DL1590-6401-8), level of C3, level of C4 and level of immunoglobulins were also recorded and analysed. ANA was detected by indirect immunofluorescence using Human epithelial type 2 (HEp-2) cells as the substrate (ANA; EUROIMMUN, FA1510-1010-1) and was defined as positive in titre $\geq 1: 100$. Anti-dsDNA antibody was detected by immunoblotting (EUROIMMUN, DL1590-6401-8), and immunofluorescent test (EUROIMMUN, FA 1572-1005) was used for verification when positive.

\section{Statistical analysis}

Data are shown as mean with SD or median with 25th75th percentiles (Q25-Q75) based on the means of distribution. Categorical data are shown as number with percentages. Differences in continuous data between groups were evaluated by either Student's t-test or MannWhitney U test. Differences in binary data between groups were evaluated by $\chi^{2}$ test or Fisher's exact test. Univariable and multivariable Cox regression analyses for CTD-free survival were performed to identify potential factors associated with risk of CTD, and HRs with 95\% CIs were calculated. Based on the results of multivariate Cox regression analyses, a CTD risk prediction model was established, and a predictive nomogram for CTD development was further constructed using the 'rms' package in R (V.3.6.1; $\mathrm{R}$ Foundation). Analyses were performed using STATA V.12.0. $\mathrm{P}<0.05$ was considered statistically significant. All authors had access to primary clinical data.

\section{Meta-analysis}

Both medical subject headings and text words for the terms 'primary immune thrombocytopenia' and 'antinuclear antibody' were combined during literature search in PubMed, Embase and Cochrane Library without any restrictions prior to 20 January 2020. Studies were included when the risk of CTD development in patients with primary ITP during follow-up was analysed and excluded if they were case reports, reviews or studies without sufficient data for data synthesis. Random-effect or fixed-effect meta-analysis was performed to pool the risk ratio (RR) with $95 \% \mathrm{CI}$ on the association between 
ANA positivity and risk of CTD among patients with primary ITP. Heterogeneity across studies was checked by $\mathrm{I}^{2}$ test, and an $\mathrm{I}^{2}$ more than $50 \%$ suggested high heterogeneity. Subgroup analyses were conducted according to risk of developing SLE among patients with ITP. All analyses were performed by STATA V.12.0. $\mathrm{P}<0.05$ was considered statistically significant. The registration ID in the international prospective register of systematic reviews (PROSPERO) is CRD42020158541.

\section{RESULTS}

\section{Characteristics of ANA-positive patients with primary ITP}

A total of 586 patients with primary ITP were successfully followed up and analysed. ANA was positive in 125 $(21.33 \%)$ patients with primary ITP included in this study. The baseline characteristics at the time of the first established primary ITP diagnosis of ANA-positive and ANAnegative patients with primary ITP are shown in table 1 . The mean follow-up time for the ANA-positive group and the ANA-negative group was 33 (18-54) months and 38 (19-57) months, respectively (table 1). No significant differences in age and sex were observed. Platelet level at diagnosis was lower in the ANA-positive group $(p=0.015$; table 1). ENA positivity in the ANA-positive and in the ANA-negative group was $39.67 \%$ and $16.93 \%$, respectively. Among ENA, anti-Sjogren's syndrome A antibody (anti-SSA) and anti-Ro52 were the main autoantibodies that were positive in patients with primary ITP. Drugs including intravenous immunoglobulin, rituximab, hydroxychloroquine (HCQ) and ciclosporin A (CsA) were used in a subset of patients with GC as initial therapy. As some ANA-positive patients with primary ITP were referred to rheumatologists who may have a different opinion from haematologists in managing patients, immunosuppressive drugs were used more slightly in the ANA-positive group $(\mathrm{p}=0.049$; table 1$)$. During follow-up, more immunosuppressive drugs especially CsA were used in ANA-positive patients with ITP, indicating that immunosuppressive drugs were needed in the ANA-positive group to avoid high-dose or long-time GC use. No significant difference was detected in response to GC therapy and chronicity (table 1 ), but a significant difference was found in the percentage of CTD development $(p<0.001)$.

\section{Risk of CTD in ANA-positive patients with primary ITP}

Among ANA-positive patients with primary ITP, 19 (15.20\%) developed CTD during follow-up, while 9 $(1.95 \%)$ in the ANA-negative group developed CTD (table 2). The characteristics of all patients with primary ITP who developed CTD are listed in online supplemental table 2. The HR of CTD in ANA-positive patients with primary ITP was 8.77 (95\% CI 3.97 to $19.40, \mathrm{p}<0.001)$. The mean time from the diagnosis of ANA-positive ITP to the development of CTD was 20.5 (10.03-30.97) months, while it was 19.2 (9.49-28.91) months from the diagnosis of ANA-positive ITP to the development of SLE. The Kaplan-Meier curve showed that the risk of CTD was significantly higher in the ANA-positive ITP group than in the ANA-negative group (log-rank $\mathrm{p}<0.001$ ) (figure $1 \mathrm{~A}$ ). CTDs that developed in patients with primary ITP included SLE, pSS, APS and UCTD. Five patients in the ANA-positive group developed SLE (5 of 125, 4.00\%), significantly higher than in the ANA-negative group ( 0 of $461,0 \%$ ) (table 2). Among the ANA-negative primary ITP group, positive ENA was detected in two patients, which might be a factor affecting CTD development (online supplemental table 2). We further classified patients with primary ITP into four groups: ANA-positive and ENApositive group (ANA+ENA+), ANA-positive and ENAnegative group (ANA+ENA-), ANA-negative and ENApositive group (ANA-ENA+), and ANA-negative and ENA-negative group (ANA-ENA-) (table 3). The risk of CTD in ANA+ENA- $(\mathrm{p}<0.001)$, ANA-ENA+ $(\mathrm{p}<0.001)$ and ANA+ENA+ $(p<0.001)$ groups were all significantly higher than in the ANA-ENA- group (figure 1B).

As not all ANA-positive patients with primary ITP developed CTD during follow-up, we further analysed the characteristics of ANA-positive patients with primary ITP who finally developed CTD (table 4). Compared with ANApositive patients with primary ITP who did not develop CTD, the level of C3 at baseline was significantly lower in those who developed CTD during follow-up $(\mathrm{p}=0.002)$. No significant differences were detected in other parameters such as C4, ANA pattern and immunoglobulin levels (table 4).

\section{Risk factors associated with CTD development in patients with primary ITP}

To investigate other factors besides positive ANA which may relate to increased risk of CTD development, Cox regression analyses were conducted. Univariate Cox regression analysis demonstrated that ANA positivity ( $\mathrm{HR}=8.77,95 \%$ CI 3.97 to $19.40, \mathrm{p}<0.001)$, ENA positivity $(\mathrm{HR}=6.55,95 \%$ CI 3.00 to $14.32, \mathrm{p}<0.001)$, anti-SSA positivity (HR=7.04, 95\% CI 3.29 to $15.06, \mathrm{p}<0.001)$, anti-Ro52 (HR=4.15, 95\% CI 1.86 to $9.26, \mathrm{p}<0.001)$ and C3 $(\mathrm{HR}=0.10,95 \%$ CI 0.02 to $0.69, \mathrm{p}=0.019)$ were significantly associated with increased risk of CTD in primary ITP (table 5). In the multivariate Cox regression analysis adjusting for confounding factors including age, sex, C3 and ENA positivity, ANA positivity was significantly associated with risk of CTD (HR=6.15, 95\% CI 2.66 to 14.23, $\mathrm{p}<0.001$; table 5). After adjusting for confounding factors including age, sex, C3 and ANA positivity, ENA positivity was significantly associated with risk of CTD $(\mathrm{HR}=3.97,95 \% \mathrm{CI} 1.75$ to $9.04, \mathrm{p}=0.001)$. After adjusting for confounding factors including age, sex, C3, ANA positivity and anti-SSB positivity, anti-SSA positivity was significantly associated with risk of CTD (HR=3.43, 95\% CI 1.49 to $7.86, \mathrm{p}=0.004$ ).

Based on the results of Cox regression analyses, a CTD risk score was established with three variables, namely ANA, anti-SSA and C3, and the CTD risk score was calculated using the following formula: CTD risk score $=1.85 \times \mathrm{ANA}+1.26 \times$ anti-SSA $-1.27 \times \mathrm{C} 3$. Using the 
Table 1 Characteristics of the study population

\begin{tabular}{|c|c|c|c|}
\hline & ANA-positive ( $n=125)$ & ANA-negative $(n=461)$ & $P$ value \\
\hline \multicolumn{4}{|l|}{ Demographic characteristics } \\
\hline Age (years) & $33.00(26.00-55.50)$ & $33.00(26.50-47.50)$ & 0.469 \\
\hline Gender (female, \%) & $102 / 125(81.60)$ & $342 / 461$ (74.19) & 0.086 \\
\hline Follow-up time (months) & $33.00(18.00-54.00)$ & $38.00(19.00-57.00)$ & 0.189 \\
\hline Bleeding score & $3.3 \pm 1.6$ & $2.8 \pm 2.0$ & 0.081 \\
\hline \multicolumn{4}{|l|}{ Baseline clinical features } \\
\hline Platelet count, $\times 10^{9} / \mathrm{L}$ & $34.00(10.00-64.75)$ & $46.00(17.25-79.00)$ & 0.015 \\
\hline Leucocyte, $\times 10^{9} / \mathrm{L}$ & $6.47(4.89-8.53)$ & $6.85(5.30-9.16)$ & 0.196 \\
\hline Neutrophils, $\times 10^{9} / \mathrm{L}$ & $4.46(3.09-6.78)$ & $4.52(3.19-7.11)$ & 0.744 \\
\hline Lymphocytes, $\times 10^{9} / \mathrm{L}$ & $1.60(1.15-2.15)$ & $1.60(1.15-2.20)$ & 0.725 \\
\hline Haemoglobin, g/L & $125.00(114.00-137.00)$ & $127.00(114.00-140.00)$ & 0.190 \\
\hline Sedimentation rate, $\mathrm{mm} /$ hour & $21.00(7.75-31.75)$ & $14.00(7.00-27.50)$ & 0.254 \\
\hline $\mathrm{C}$ reactive protein, $\mathrm{mg} / \mathrm{L}$ & $1.19(0.74-4.20)$ & $1.21(0.73-3.19)$ & 0.944 \\
\hline Anti-PAlgG positivity, n (\%) & $23 / 50(46.00)$ & 59/166 (35.54) & 0.182 \\
\hline ENA positivity, n (\%) & 48/121 (39.67) & $76 / 449$ (16.93) & $<0.001$ \\
\hline Anti-SSA positivity & 28/121 (23.14) & $31 / 449(6.90)$ & $<0.001$ \\
\hline Anti-Ro52 positivity & 26/121 (21.49) & $42 / 449$ (9.35) & $<0.001$ \\
\hline Anti-SSB positivity & 3/121 (2.48) & $3 / 449(0.67)$ & 0.218 \\
\hline $\mathrm{C} 3, \mathrm{mg} / \mathrm{dL}$ & $1.01(0.85-1.15)$ & $1.02(0.89-1.17)$ & 0.198 \\
\hline $\mathrm{C} 4, \mathrm{mg} / \mathrm{dL}$ & $0.21(0.17-0.27)$ & $0.22(0.17-0.28)$ & 0.390 \\
\hline $\lg A, g / L$ & $2.20(1.69-2.84)$ & $1.99(1.53-2.54)$ & 0.026 \\
\hline $\operatorname{lgG}, g / L$ & $13.95(11.70-15.78)$ & $12.10(10.33-14.38)$ & $<0.001$ \\
\hline $\lg M, g / L$ & $1.12(0.82-1.63)$ & $1.22(0.84-1.71)$ & 0.216 \\
\hline \multicolumn{4}{|l|}{ Initial treatment, $\mathrm{n}(\%)$} \\
\hline Glucocorticoids & 99/100 (99.00) & $251 / 253(99.21)$ & 1.000 \\
\hline Other drugs & $11 / 100(11.00)$ & $13 / 253(5.14)$ & 0.049 \\
\hline \multicolumn{4}{|c|}{ Therapeutic measures used during follow-up } \\
\hline Hydroxychloroquine, n (\%) & 6/99 (6.06) & $7 / 253(2.77)$ & 0.204 \\
\hline Ciclosporin, n (\%) & $7 / 99(7.07)$ & $4 / 253(1.58)$ & 0.014 \\
\hline Tacrolimus, n (\%) & $1 / 99(1.01)$ & $1 / 253(0.40)$ & 0.484 \\
\hline Rituximab, n (\%) & 3/99 (3.03) & $5 / 253(1.98)$ & 0.691 \\
\hline IVIG, n (\%) & 3/99 (3.03) & 4/253 (1.58) & 0.407 \\
\hline Splenectomy, n (\%) & $3 / 121(2.48)$ & $3 / 453(0.66)$ & 0.112 \\
\hline Drug-free time & $1.32(1.01-1.62)$ & $1.09(0.94-1.25)$ & 0.165 \\
\hline \multicolumn{4}{|l|}{ Prognosis, n (\%) } \\
\hline Chronic ITP & 58/99 (58.59) & 195/313 (62.30) & 0.508 \\
\hline Resistant to steroids & 16/93 (17.20) & 26/218 (11.93) & 0.212 \\
\hline CTD development & 19/125 (15.20) & 9/461 (1.95) & $<0.001$ \\
\hline
\end{tabular}

Anti-SSA, anti-Sjogren's syndrome A antibody; anti-SSB, anti-Sjogren's syndrome B antibody; CTD, connective tissue disease; ENA, extractable nuclear antigen antibodies; ITP, immune thrombocytopaenia; IVIG, intravenous immunoglobulin; PAlgG, platelet-associated IgG.

variables associated with increased risk of CTD development in patients with primary ITP, we constructed a nomogram for predicting the 3-year and 5-year probability of CTD-free survival among patients with primary ITP (figure 2).
Meta-analysis of risk of CTD in ANA-positive primary ITP

As studies about the association of positive ANA and CTD development were controversial, we further performed a meta-analysis to evaluate the overall risk of CTD development in ANA-positive primary ITP. The flow chart of the 
Table 2 Risk of CTD occurrence in ANA-positive patients with primary ITP

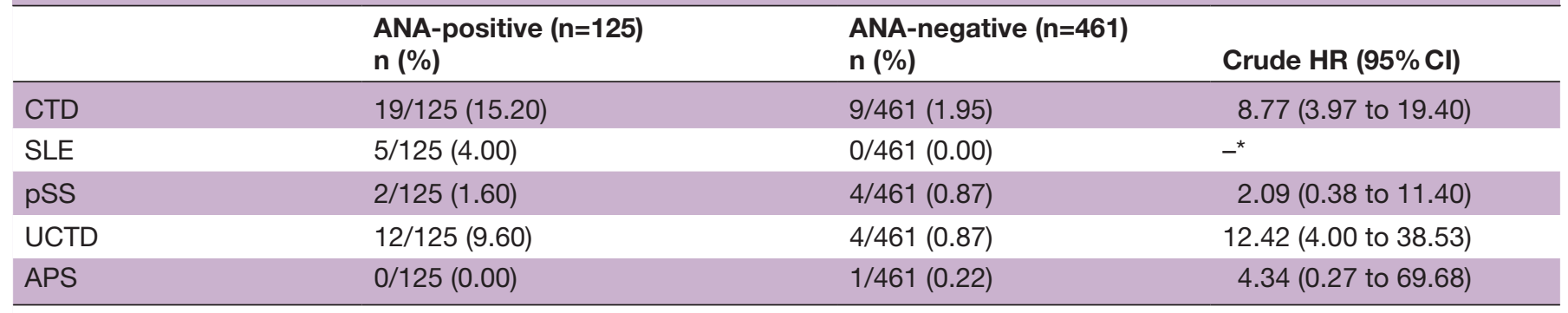

*Data were unable to be calculated.

APS, antiphospholipid syndrome; CTD, connective tissue disease; pSS, primary Sjogren's syndrome; UCTD, undifferentiated connective tissue disease.

literature selection process for this meta-analysis is shown in online supplemental figure 2. A total of 1064 studies were obtained from PubMed $(\mathrm{n}=435)$, Embase $(\mathrm{n}=627)$ and Cochrane Library $(\mathrm{n}=2)$. After screening studies by inclusion criteria and removing duplicates, 12 eligible studies comprising 1577 primary ITP cases (244 ANApositive primary ITP and 1333 ANA-negative primary ITP, respectively) were finally adopted. ${ }^{13-24}$ The characteristics of the included studies are shown in online supplemental table 3. The follow-up outcomes of the present study were also included in the meta-analysis. When pooling data from these studies (369 ANA-positive primary ITP and 1794 ANA-negative primary ITP, respectively), the rate of positive ANA in the total number of patients with ITP was $17.06 \%$ (369 of 2163). A significantly increased risk of CTD development among ANA-positive patients with primary ITP was observed ( $R R=12.43,95 \%$ CI 7.91 to $19.55, \mathrm{p}<0.00001 ; \mathrm{I}^{2}=35 \%$ ) (figure $3 \mathrm{~A}$ ). Also, ANA-positive patients with primary ITP were at higher risk of developing SLE (RR=30.41, 95\% CI 13.23 to $69.86, \mathrm{p}<0.00001$; $\mathrm{I}^{2}=0 \%$ ) (figure $3 \mathrm{~B}$ ).
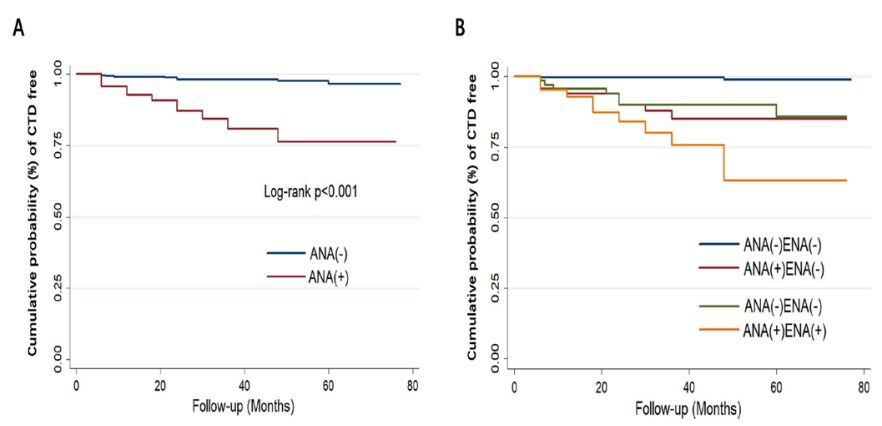

Figure 1

Figure 1 Cumulative probability of CTD-free survival of patients with primary ITP stratified by ANA or ENA. (A) Cumulative probability of CTD-free survival of patients with primary ITP with and without ANA. (B) Cumulative probability of CTD-free survival of patients with primary ITP stratified by both ANA and ENA. CTD, connective tissue disease; ENA, extractable nuclear antigen antibodies; ITP, immune thrombocytopaenia.

\section{DISCUSSION}

To the best of our knowledge, this study is the largest cohort study to assess the risk of CTD development in ANA-positive primary ITP. The results of this study confirmed that ANA-positive patients with primary ITP were at 21.6 times higher risk of new-onset CTD and 48 times higher risk of new-onset SLE compared with other patients with primary ITP, supporting that ANA-positive primary ITP is a distinct entity from other patients with primary ITP with regard to the risk of developing CTDs.

Thrombocytopaenia is a common clinical manifestation of CTDs, especially SLE, and is one of the haematological criteria for SLE diagnosis. ${ }^{25}$ In about $5 \%$ of patients with SLE, isolated thrombocytopaenia can be the first clinical manifestation, ${ }^{22}$ and it has been proven that patients with primary ITP are at increased risk of SLE development. ${ }^{26}$ Thus, patients with thrombocytopaenia with positive ANA may sometimes resemble the preclinical stage of SLE. As the treatment principles for primary ITP are quite different from SLE as well as other CTDs, ${ }^{27}$ and it has been well recognised that thrombocytopaenia secondary to SLE is typically lengthy and is often characterised by relapses during GC tapering, ${ }^{28}$ the best treatment strategy and what should be done for ANA-positive patients with ITP are key questions remaining to be answered by both haematologists and rheumatologists. HCQ was reported to delay incomplete lupus erythematosus in its transition to SLE. ${ }^{29}$ Incomplete lupus erythematosus is an early stage of SLE characterised by the presence of ANA and other clinical or laboratory findings but does no not satisfy the classification criteria for SLE. ${ }^{30}$ ITP with positive ANA might be an incomplete lupus erythematosus, and this subset of patients might benefit from the use of HCQ. However, more research are needed. This study demonstrated that ANA-positive patients with ITP are at the highest risk of developing CTD in the first 4 years after their first established ITP diagnosis, and then the risk starts to decline. We developed a promising easy-to-use tool to score the risk of CTD using ANA, anti-SSA and C3 results. Therefore, it is worthy of attention from haematologists when primary ITP presents any rheumatic manifestations during the first 4 years of follow-up, and a referral to a rheumatologist is needed when CTDs are suspected. 
Table 3 Risk of CTD occurrence in autoantibody-positive patients with primary ITP

\begin{tabular}{lccll}
\hline Groups & $\mathbf{n}$ & Cases, $\mathbf{n}(\%)$ & Crude HR $\mathbf{~ 9 5 \% ~ C l ) ~}$ & P value \\
\hline ANA-/ENA- & 373 & $2(0.5)$ & Reference & - \\
ANA+/ENA- & 73 & $8(11.0)$ & $21.9(4.7$ to 103.3$)$ & $<0.001$ \\
ANA-/ENA+ & 76 & $7(9.2)$ & $16.8(3.5$ to 81.3$)$ & $<0.001$ \\
ANA+/ENA+ & 48 & $10(20.8)$ & $47.7(10.4$ to 218.2$)$ & $<0.001$ \\
\hline
\end{tabular}

CTD, connective tissue disease; ENA, extractable nuclear antigen antibodies.

Large number of studies have demonstrated that patients with primary ITP are a heterogeneous population, ${ }^{2}$ but little is known about the subclassification of primary ITP clinically. The present study showed the positivity of ANA was high in adult patients with primary ITP (125 of 586, 21.33\%), similar to the overall rate of ANA positivity in studies included in the meta-analysis (369 of $2163,17.06 \%)$. Debate has been raised in whether patients with positive ANA primary ITP should be distinguished from other patients with primary ITP, as CTD development risk might be increased in patients with positive ANA. However, they were still considered as primary ITP based on several studies that concluded that ANA was not associated with high -risk development of SLE as well as other CTDs. ${ }^{31} 32$ Our study confirmed the increased risk of CTD development in ANA-positive primary ITP and showed that the occurrence rate of CTD was low in ANApositive primary ITP (19 of 125, 15.20\%). Owing to the low occurrence rate of CTD during follow-up, a significant association might be difficult to be identified in studies with relatively small sample size, which might contribute to differences in the results of the studies. Heterogeneity in cut-offs for positive ANA, follow-up period and race might also contribute to the different results.

Table 4 Characteristics of ANA-positive patients with primary ITP who developed CTD

\begin{tabular}{|c|c|c|c|}
\hline & Developed CTD (n=19) & Did not develop CTD $(n=106)$ & $P$ value \\
\hline \multicolumn{4}{|l|}{ Basic characteristics } \\
\hline Age, years & $34.00(26.00-45.00)$ & $32.50(25.75-56.50)$ & 0.754 \\
\hline Female/male (female, \%) & $12 / 7(63.16)$ & $90 / 16(84.91)$ & 0.053 \\
\hline Mean length of follow-up (months) & $38.42 \pm 18.46$ & $34.80 \pm 22.35$ & 0.507 \\
\hline Bleeding score & $4.1 \pm 1.5$ & $3.1 \pm 1.6$ & 0.169 \\
\hline \multicolumn{4}{|l|}{ Clinical features } \\
\hline Platelet count, $\times 10^{9} / \mathrm{L}$ & $43.00(10.00-61.00)$ & $32.00(9.50-66.00)$ & 0.983 \\
\hline Leucocyte, $\times 10^{9} / \mathrm{L}$ & $5.85(4.18-8.16)$ & $6.62(5.03-8.74)$ & 0.261 \\
\hline Neutrophils, $\times 10^{9} / \mathrm{L}$ & $3.75(2.10-6.60)$ & $4.65(3.18-6.83)$ & 0.287 \\
\hline Lymphocytes, $\times 10^{9} / \mathrm{L}$ & $1.36(0.90-2.15)$ & $1.61(1.17-2.24)$ & 0.203 \\
\hline Haemoglobin, g/L & $125.00(109.00-146.00)$ & $125.00(114.00-137.00)$ & 0.901 \\
\hline Sedimentation rate, $\mathrm{mm} / \mathrm{hour}$ & $17.75 \pm 16.19$ & $24.95 \pm 17.75$ & 0.461 \\
\hline $\mathrm{C}$ reactive protein, $\mathrm{mg} / \mathrm{L}$ & $2.11(0.78-3.56)$ & $1.09(0.69-4.61)$ & 0.303 \\
\hline Anti-PAlgG positivity, n (\%) & $3 / 7(42.86)$ & $20 / 43(46.51)$ & 1.000 \\
\hline ANA pattern (speckled pattern), $\mathrm{n}(\%)$ & $14 / 18(77.78)$ & $67 / 94(71.28)$ & 0.572 \\
\hline ENA positivity, n (\%) & 10/18 (55.56) & 38/103 (36.89) & 0.135 \\
\hline Anti-SSA positivity & $7 / 18(38.89)$ & 21/103 (20.39) & 0.157 \\
\hline Anti-Ro52 positivity & $5 / 18(27.78)$ & 21/103 (20.39) & 0.694 \\
\hline Anti-SSB positivity & $0 / 18(0.00)$ & $3 / 103(2.91)$ & 1.000 \\
\hline $\mathrm{C} 3, \mathrm{mg} / \mathrm{dL}$ & $0.81(0.73-1.02)$ & $1.03(0.87-1.20)$ & 0.002 \\
\hline $\mathrm{C} 4, \mathrm{mg} / \mathrm{dL}$ & $0.17(0.14-0.27)$ & $0.21(0.17-0.27)$ & 0.177 \\
\hline $\lg A, g / L$ & $1.81(1.42-2.93)$ & 2.27 (1.72-2.86) & 0.250 \\
\hline $\lg G, g / L$ & $13.90(12.03-16.73)$ & $13.95(11.65-15.60)$ & 0.522 \\
\hline $\lg M, g / L$ & $0.95(0.72-1.13)$ & $1.15(0.84-1.73)$ & 0.081 \\
\hline
\end{tabular}

Anti-SSA, anti-Sjogren's syndrome A antibody; anti-SSB, anti-Sjogren's syndrome B antibody; CTD, connective tissue disease; ENA, extractable nuclear antigen antibodies; ITP, immune thrombocytopaenia; PAlgG, platelet-associated IgG. 
Table 5 Cox regression analyses of risk of CTD in patients with primary ITP

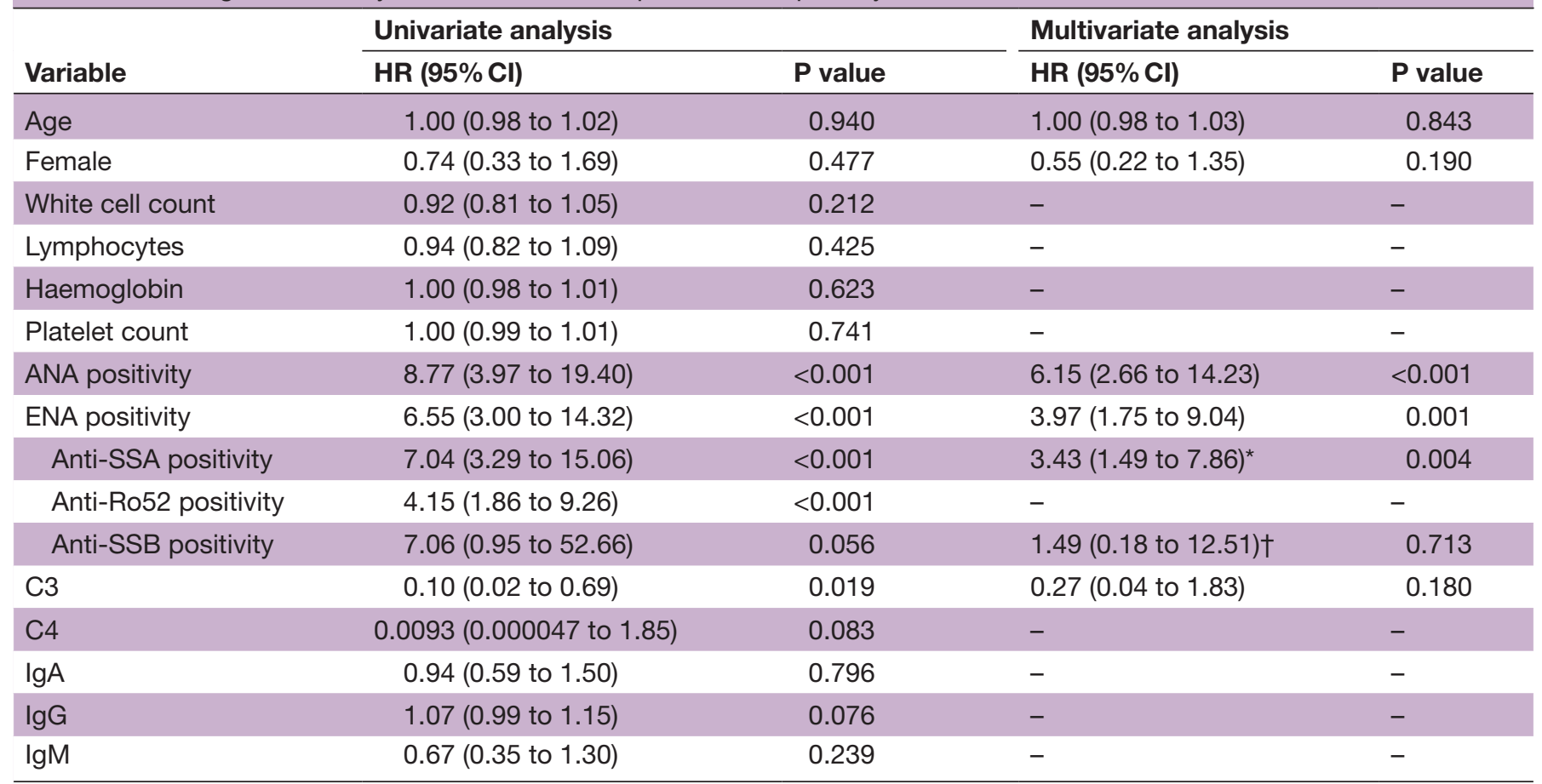

${ }^{*}$ Outcome for anti-SSA was adjusted for ANA, C3, anti-SSB, age and gender. †Outcome for anti-SSB was adjusted for ANA, C3, anti-SSA, age and gender.

Anti-SSA, anti-Sjogren's syndrome A antibody; anti-SSB, anti-Sjogren's syndrome B antibody; ENA, extractable nuclear antigen antibodies.

Although it has been indicated by previous studies that ANA-positive patients with primary ITP were at high risk

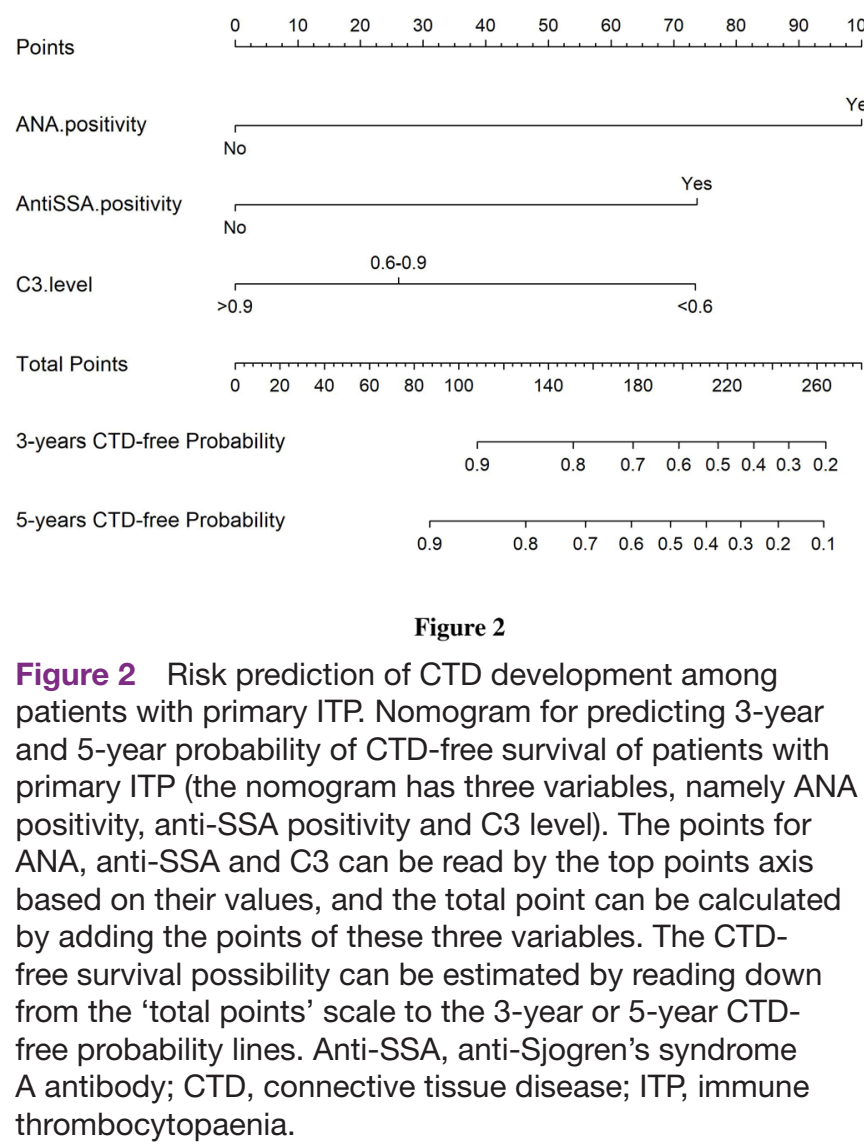

of CTD development, it remains unclear which subgroup of ANA-positive patients with primary ITP were especially at high risk of CTD or SLE development. In the present study, we found that C3 level was significantly lower in ANA-positive patients who developed CTD compared with those who did not develop CTD, and among the autoantibodies anti-SSA and anti-Ro52 were the most

A

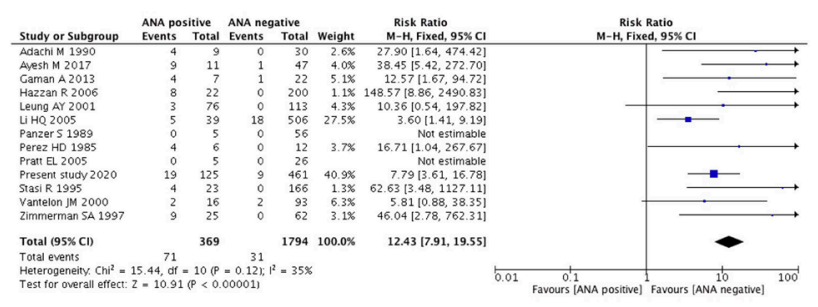

B

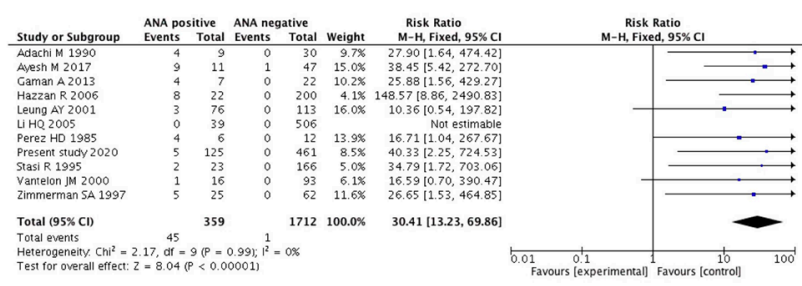

Figure 3

Figure 3 Forest plots of the meta-analysis. (A) Forest plot of the meta-analysis of risk of CTD among patients with primary ITP with positive ANA. (B) Forest plot of the metaanalysis of risk of SLE among patients with primary ITP with positive ANA. CTD, connective tissue diseases; ITP, immune thrombocytopaenia; M-H, Mantel-Haenszel. 
common autoantibodies present in patients with primary ITP, indicating the possible role of C3, anti-SSA and antiRo52 in linking thrombocytopenia and systemic autoimmune diseases.

Complement is part of the innate immune system and is the main effector mechanism of antibody-mediated immunity. Activation of complement has been proven to be involved in many systemic autoimmune diseases such as SLE, pSS and APS and is associated with disease activity. ${ }^{33}$ As shown in other systemic diseases, low C3 level in ANA-positive patients with primary ITP who developed CTD indicates consumption of C3 and activation of complement system. How C3 was involved in the association of thrombocytopaenia and systemic autoimmune disease still needs to be investigated.

Autoantibodies to SSA/Ro are traditionally considered to be associated with CTDs such as SLE and $\mathrm{pSS},{ }^{34}$ which can precede other SLE-associated autoantibodies such as anti-dsDNA and are present on an average time of 3.4 years before the diagnosis of SLE.$^{35}$ Two different targeted proteins of SSA/Ro antibody with distinct biochemical and immunological functions were later described as Ro60 $(60 \mathrm{kDa})$ and Ro52 $(52 \mathrm{kDa})$, and the targeted protein of SSA only refers to antigen Ro60 at present. ${ }^{36}$ Although no studies have revealed the association of anti-SSA or antiRo52 with thrombocytopaenia, their potential role in the pathogenesis of thrombocytopaenia was indicated by a case report. ${ }^{37}$ The present study found anti-SSA and antiRo52 were the most common autoantibodies among ENA present in patients with primary ITP and were risk factors for CTD development. Further studies focusing on the role of SSA and Ro52 in the pathogenesis of thrombocytopenia might help us have a better understanding of ITP.

There were several limitations to this study. First, this study used a retrospective design. In our study, almost a third of patients failed to be followed up and many of them might have had adverse outcomes, thus skewing the results. Second, some new manifestations of SLE such as glomerulonephritis might be underestimated just by telephone interview during follow-up before more rheumatic manifestations occurs, which might under-rate the CTDs in primary ITP. Third, the findings of our study, especially the predictive nomogram, need to be validated in a second cohort. Fourth, only adult patients were included in this study, while in paediatric setting the situation may be different and the results should be interpreted carefully. Finally, there were some limitations that might impact the interpretation of the results of this meta-analysis. Several studies lacked data on patients developing autoimmune diseases among patients with primary ITP with negative ANA, ${ }^{15} 31323839$ which were excluded from this metaanalysis but might have an impact on the results.

In conclusion, our results demonstrated that patients with primary ITP with positive ANA were at significantly increased risk of developing CTDs, especially SLE, compared with other patients with primary ITP, indicating that primary ITP with positive ANA might be a clinical entity distinct from other primary ITP at least in the aspect of prognosis. Studies on ANA-positive primary ITP might help us a lot in better management of primary ITP and achieving an early diagnosis of SLE, and moreover may open a new window to understanding the early pathogenesis course of SLE.

Acknowledgements The authors would like to thank the authors of the included studies.

Contributors YL and GS performed the study design. YL, SC and GY analysed the data and wrote the manuscript. FD, PR, PW, HQ, JL and BW collected and analysed the data. All authors approved the final manuscript.

Funding The work was supported by the Natural Science Foundation of China grants to YL (81971496) and GS (81971536) and the Fujian Science and Technology Project 2020J011250 to YL and 2020J05309 to SC.

Competing interests None declared.

Patient consent for publication Not applicable.

Ethics approval This study was approved by the Clinical Research Ethics Committee of the First Affiliated Hospital of Xiamen University (KY2016-001). Provenance and peer review Not commissioned; externally peer reviewed. Data availability statement Data are available upon reasonable request. The data are available from the corresponding author, gshi@xmu.edu.cn.

Supplemental material This content has been supplied by the author(s). It has not been vetted by BMJ Publishing Group Limited (BMJ) and may not have been peer-reviewed. Any opinions or recommendations discussed are solely those of the author(s) and are not endorsed by BMJ. BMJ disclaims all liability and responsibility arising from any reliance placed on the content. Where the content includes any translated material, BMJ does not warrant the accuracy and reliability of the translations (including but not limited to local regulations, clinical guidelines, terminology, drug names and drug dosages), and is not responsible for any error and/or omissions arising from translation and adaptation or otherwise.

Open access This is an open access article distributed in accordance with the Creative Commons Attribution Non Commercial (CC BY-NC 4.0) license, which permits others to distribute, remix, adapt, build upon this work non-commercially, and license their derivative works on different terms, provided the original work is properly cited, appropriate credit is given, any changes made indicated, and the use is non-commercial. See: http://creativecommons.org/licenses/by-nc/4.0/.

\section{ORCID iDs}

Yuan Liu http://orcid.org/0000-0003-2668-0350

Guixiu Shi http://orcid.org/0000-0003-4044-3394

\section{REFERENCES}

1 Rodeghiero F, Stasi R, Gernsheimer T, et al. Standardization of terminology, definitions and outcome criteria in immune thrombocytopenic purpura of adults and children: report from an international working group. Blood 2009;113:2386-93.

2 Cines DB, Bussel JB, Liebman HA, et al. The ITP syndrome: pathogenic and clinical diversity. Blood 2009;113:6511-21.

3 Lambert MP, Gernsheimer TB. Clinical updates in adult immune thrombocytopenia. Blood 2017;129:2829-35.

4 Marmont AM. Antinuclear antibody (ANA)-positive thrombocytopenia: primary, but with a difference. Blood 2009;114:2003-4. author reply 04.

5 Neunert C, Lim W, Crowther M, et al. The American Society of hematology 2011 evidence-based practice guideline for immune thrombocytopenia. Blood 2011;117:4190-207.

6 Tan EM, Cohen AS, Fries JF, et al. The 1982 revised criteria for the classification of systemic lupus erythematosus. Arthritis Rheum 1982;25:1271-7.

7 Shiboski SC, Shiboski CH, Criswell LA, et al. American College of rheumatology classification criteria for Sjögren's syndrome: a data-driven, expert consensus approach in the Sjögren's international collaborative clinical alliance cohort. Arthritis Care Res 2012;64:475-87.

8 Wilson WA, Gharavi AE, Koike T, et al. International consensus statement on preliminary classification criteria for definite antiphospholipid syndrome: report of an international workshop. Arthritis Rheum 1999;42:1309-11. 
9 van den Hoogen F, Khanna D, Fransen J. Classification criteria for systemic sclerosis: an ACR-EULAR collaborative initiative. Arthritis Rheum 2013;65:2737.

10 Bohan A, Peter JB. Polymyositis and dermatomyositis (first of two parts). N Engl J Med 1975;292:344-7.

11 Mosca M, Neri R, Bombardieri S. Undifferentiated connective tissue diseases (UCTD): a review of the literature and a proposal for preliminary classification criteria. Clin Exp Rheumatol 1999;17:615-20.

12 Page LK, Psaila B, Provan D, et al. The immune thrombocytopenic purpura (ITP) bleeding score: assessment of bleeding in patients with ITP. Br J Haematol 2007;138:245-8.

13 Panzer S, Penner E, Graninger W, et al. Antinuclear antibodies in patients with chronic idiopathic autoimmune thrombocytopenia followed 2-30 years. Am J Hematol 1989;32:100-3.

14 Perez HD, Katler E, Embury S. Idiopathic thrombocytopenic purpura with high-titer, speckled pattern antinuclear antibodies: possible marker for systemic lupus erythematosus. Arthritis Rheum 1985;28:596-7.

15 Adachi M, Mita S, Obana M, et al. Thrombocytopenia subsequently develops systemic lupus erythematosus--can anti-SS-A antibody predict the next event? Jpn J Med 1990;29:481-6.

16 Stasi R, Stipa E, Masi M, et al. Long-term observation of 208 adults with chronic idiopathic thrombocytopenic purpura. Am J Med 1995;98:436-42.

17 Zimmerman SA, Ware RE. Clinical significance of the antinuclear antibody test in selected children with idiopathic thrombocytopenic purpura. J Pediatr Hematol Oncol 1997;19:297-303.

18 Vantelon JM, Godeau B, André C, et al. Screening for autoimmune markers is unnecessary during follow-up of adults with autoimmune thrombocytopenic purpura and NO autoimmune markers at onset. Thromb Haemost 2000;83:42-5.

19 Leung AY, Chim CS, Kwong YL, et al. Clinicopathologic and prognostic features of chronic idiopathic thrombocytopenic purpura in adult Chinese patients: an analysis of 220 cases. Ann Hematol 2001;80:384-6.

20 Pratt EL, Tarantino MD, Wagner D, et al. Prevalence of elevated antithyroid antibodies and antinuclear antibodies in children with immune thrombocytopenic purpura. Am J Hematol 2005;79:175-9.

$21 \mathrm{Li} \mathrm{H}$-qiang, Zhang L, Zhao H, et al. Chronic idiopathic thrombocytopenic purpura in adult Chinese patients: a retrospective single-centered analysis of 1791 cases. Chin Med J 2005;118:34-7.

22 Hazzan R, Mukamel M, Yacobovich J, et al. Risk factors for future development of systemic lupus erythematosus in children with idiopathic thrombocytopenic purpura. Pediatr Blood Cancer 2006;47:657-9.

23 Gaman A, Gaman G, Rotaru I. The antiphospholipid antibodies, a risk factor to developed antiphospholipide syndrome or systemic lupus Erythematous in the patients with immune thrombocytopenic purpura (ITP). Haematologica 2013;98:742.
24 Ayesh M, Alawneh K, Khader Y. Risk of lupus after primary immune thrombocytopenic purpura: a 14 year single center experience. Haematologica 2017;102:840-1.

25 Fayyaz A, Igoe A, Kurien BT, et al. Haematological manifestations of lupus. Lupus Sci Med 2015;2:e000078.

26 Zhu F-X, Huang J-Y, Ye Z, et al. Risk of systemic lupus erythematosus in patients with idiopathic thrombocytopenic purpura: a population-based cohort study. Ann Rheum Dis 2020;79:793-9.

27 Fanouriakis A, Kostopoulou M, Alunno A, et al. 2019 update of the EULAR recommendations for the management of systemic lupus erythematosus. Ann Rheum Dis 2019;78:736-45.

28 Jung J-H, Soh M-S, Ahn Y-H, et al. Thrombocytopenia in systemic lupus erythematosus: clinical manifestations, treatment, and prognosis in 230 patients. Medicine 2016;95:e2818.

29 James JA, Kim-Howard XR, Bruner BF, et al. Hydroxychloroquine sulfate treatment is associated with later onset of systemic lupus erythematosus. Lupus 2007;16:401-9.

30 Olsen NJ, Karp DR. Hydroxychloroquine: an old drug with new tricks. $J$ Rheumatol 2021;48:796-8.

31 Altintas A, Ozel A, Okur N, et al. Prevalence and clinical significance of elevated antinuclear antibody test in children and adult patients with idiopathic thrombocytopenic purpura. J Thromb Thrombolysis 2007;24:163-8.

32 Kurata Y, Miyagawa S, Kosugi S, et al. High-Titer antinuclear antibodies, anti-SSA/Ro antibodies and anti-nuclear RNP antibodies in patients with idiopathic thrombocytopenic purpura. Thromb Haemost 1994;71:184-7.

33 Chen M, Daha MR, Kallenberg CGM. The complement system in systemic autoimmune disease. J Autoimmun 2010;34:J276-86.

34 Zappi E, Sontheimer R. Clinical relevance of antibodies to Ro/SS$\mathrm{A}$ and $\mathrm{La} / \mathrm{SS}-\mathrm{B}$ in subacute cutaneous lupus erythematosus and related conditions. Immunol Invest 1993;22:189-203.

35 Arbuckle MR, McClain MT, Rubertone MV, et al. Development of autoantibodies before the clinical onset of systemic lupus erythematosus. N Engl J Med 2003;349:1526-33.

36 Robbins A, Hentzien M, Toquet S, et al. Diagnostic utility of separate Anti-Ro60 and Anti-Ro52/TRIM21 antibody detection in autoimmune diseases. Front Immunol 2019;10:444.

37 Ayadi ID, Ben Hamida E, Boukhris MR, et al. Isolated anti-Ro/SSA thrombocytopenia: a rare feature of neonatal lupus. Pan Afr Med $J$ 2015;22:312.

38 Anderson MJ, Peebles CL, McMillan R, et al. Fluorescent antinuclear antibodies and anti-SS-A/Ro in patients with immune thrombocytopenia subsequently developing systemic lupus erythematosus. Ann Intern Med 1985;103:548-50.

39 Demir C, Esen R, Atmaca M, et al. Prevalence of autoantibodies related to some autoimmune disorders in patients with chronic idiopathic thrombocytopenic purpura. Clin Appl Thromb Hemost 2011;17:E114-8. 